\title{
Fun with Photons: Selective Light Induced Reactions in Solution and in Water Soluble Nano-containers
}

\author{
Anoklase Ayitou, Barry C. Pemberton, Elango Kumarasamy, Nandini Vallavoju, \\ and J. Sivaguru $\AA^{{ }^{*}}$
}

§Gramaticakis-Neumann Prize Winner 2010

\begin{abstract}
Two distinct strategies for controlling selectivity, in particular stereoselectivity in photochemical reactions are reviewed. In the first strategy, supramolecular approach using cucurbituril nano-containers in catalytic amounts is employed to control selectivity during photochemical transformations. In the second approach, a generalized methodology for carrying out light-induced transformations in solution at ambient conditions is detailed where axially chiral motifs are employed to enantiospecifically transfer the axial chirality in the reactant to point chirality in the photoproduct(s).
\end{abstract}

Keywords: Asymmetric photoreactions - Cucurbiturils · Host-Guest chemistry · Molecular chirality · Supramolecular catalysis

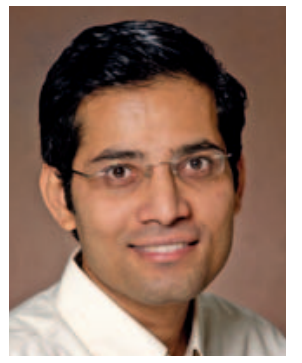

Prof.Sivaguru Jaya$\operatorname{raman}($ Siva) is a 5 th year assistant professor at the Department of Chemistry and Biochemistry, North Dakota State University, Fargo, ND, USA. He received his Bachelors degree (1996) from St. Joseph's College (Autonomous), Bharathidasan University, Trichy, India and Masters degree (1998) from IIT-Madras, Chennai, India. He received his PhD degree (2003) in chemistry from Tulane University, New Orleans under the guidance of Prof. V. Ramamurthy. He completed a post-doctoral fellowship (2003-2006) at Columbia University, NY under the mentorship of Prof. Nicholas J. Turro. Siva moved to his current position at NDSU in 2006 and established an independent research program that has gained prominence among his peers within a very short time as evidenced by the CAREER

\footnotetext{
*Correspondence: Prof. Dr. J. Sivaguru North Dakota State University Department of Chemistry and Biochemistry 1231 Albrecht Blvd.,

104H, LADD Hall, P.O. Box 6050, NDSU Dept 2735 Fargo, ND 58108-6050. USA

Tel.: +1 7012318923

Fax: +1 7012318831

E-mail: sivaguru.jayaraman@ndsu.edu
}

award from the National Science Foundation (2008). At NDSU, his research talents were recognized with the 2010 College of Science and Mathematic Award for Excellence in Research. His current research interests include Photocatalysis (supramolecular and organic photocatalysis), Light induced asymmetric transformations, Photochemistry within organized and confined media, Supramolecular photochemistry, Host-Guest Systems, Molecular recognition in chemical and biological systems and Molecular Self-Assembly.

The review article details two distinct ways of controlling selectivity, in particular stereoselectivity in photochemical reactions. The first part uses water-soluble nano-container molecules known as cucurbiturils in catalytic amounts to control selectivity while the second aspect addresses a traditional challenge of enantiocontrol in photoreactions, where molecularly chiral (axially chiral) chromophores are employed for enantiospecific photoreactions.

\section{Water-soluble Nano-containers as Supramolecular Photocatalysts}

Exploiting supramolecular interactions to alter/improve existing reactivity and/ or selectivity has been a topic of interest due to the prospect of using nano-inspired materials for harvesting light induced transformations. ${ }^{[1]}$ Additionally, the nanoconfinement imposed by macrocyclic hosts such as cyclodextrins, ${ }^{[2-4]}$ cucurbit[n] urils $^{[5-12]}$ and synthetic capsules ${ }^{[13,14]}$ has shown promise towards the development of efficient enzyme-mimetics ${ }^{[2,3]}$ for various chemical transformations. ${ }^{[12,15-17]}$ Some key factors that have to be considered before employing organized assemblies to control photoreactivity within supramolecular environments ${ }^{[18]}$ are i) available free space, ii) structural rigidity and iii) type of non-bonding interaction that develops between the host and the guest. In our opinion cucurbiturils not only satisfy the above requirements, but also are watersoluble, making them appealing from an environmental perspective.

\subsection{Cucurbit[n]urils}

Cucurbiturils ${ }^{[5-12]}$ are a family of molecular container compounds with shapes similar to that of pumpkins (botanical name cucurbitaceae $\left.{ }^{[6]}\right)$. Cucurbiturils $(\mathrm{CBs})^{[5-12]}$ feature a cavity similar to that of cyclodextrins (Scheme 1). Similar to cyclodextrins that are made of D-glucopyranosyl units, CBs are constructed of glycouril units. The number of glycouril units (denoted by CB6, CB7 and CB8) determines the available volume within cucurbiturils (CBs). The glycouril unit provides a hydrophobic pocket (nanocavity) in which the portal is made of polar carbonyl groups that allows them to bind to both polar and non-polar organic molecules. Recent efforts by various groups ${ }^{[7-12,19]}$ have opened up new opportunities for using cucurbiturils as 'nano-reaction vessels'. Cucurbit[8]urils 


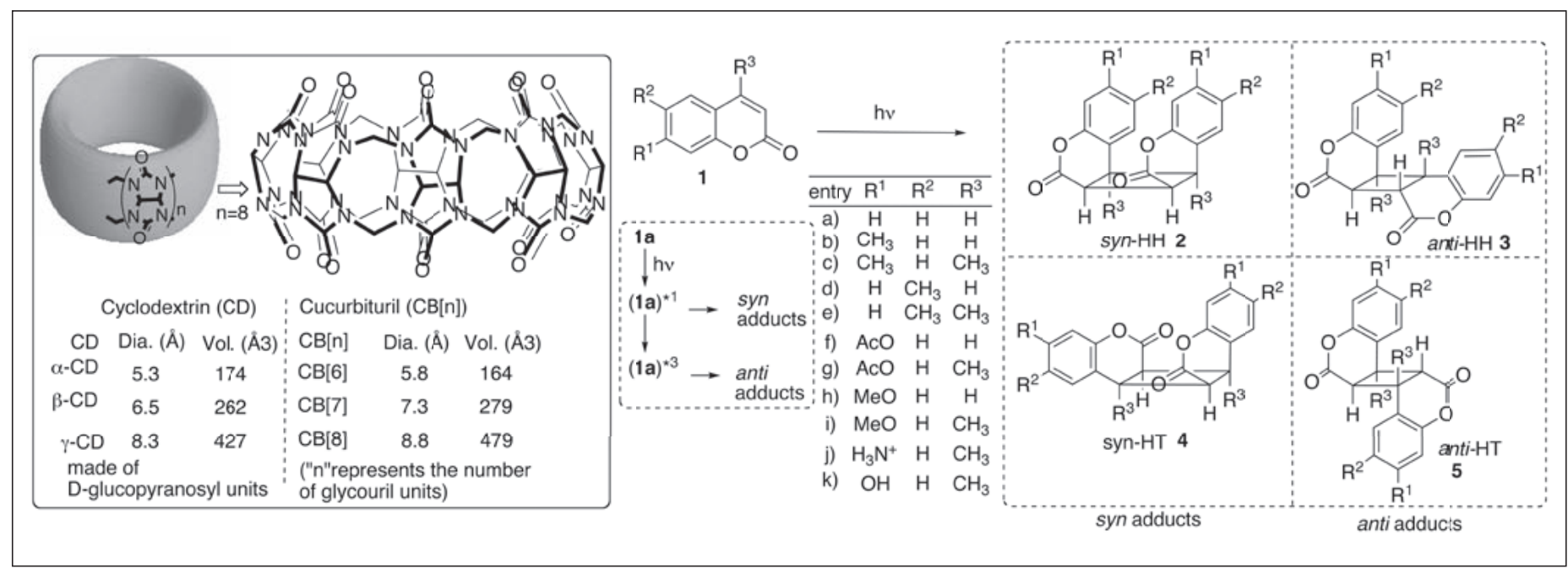

Scheme 1. Left: Structural comparison of cucurbit[n]urils (CBs) and cyclodextris (CDs); Right: Photodimerization of coumarin derivatives.

(CB[8]) attracted our attention as their cavity volume is similar to that of $\gamma$-cyclodextrins $(\gamma-\mathrm{CD})$. We chose to investigate the photodimerization of coumarin derivatives 1 (Scheme 1) within $\mathrm{CB}[8]$ as we reasoned that $\mathrm{CB}[8]$ with a similar (Scheme 1) cavity volume $\left(479 \AA^{3}\right)$ as that of $\gamma$-CD (cavity volume $427 \AA^{3}$ ), will be an ideal candidate and will most likely form 1:2 host-guest (HG) complexes, as $\gamma$-CD forms a 1:2 complex with various coumarin derivatives and other photochromophores. ${ }^{[20]}$

\subsection{Supramolecular Catalysis with CBs}

Using 'nano-cavities' to confine reactive photochromophores allows for investigation of photoreactions based on size/ shape of the nano-cavity along with the structural rigidity and non-bonding interactions that develop between the host and guest within the nano-cavity. To successfully employ $\mathrm{CB}[8]$ as a 'nano-reaction vessel' for synthetic transformations, it is critical to employ them in catalytic amounts in order to overcome a fundamental bottleneck viz., solubility of $\mathrm{CB}[8]$ in high amounts $(>0.2 \mathrm{mM})$ that is typically employed for synthetic reactions.

\subsection{Coumarin Derivatives as Molecular Probes}

Coumarin has historically been the subject of intense photochemical and spectroscopic interest mainly as a consequence of its significance in biological systems and use in various materials of commercial interest. ${ }^{[21-28]}$ Recently, we have been exploring the role of cucurbit[8]urils $(\mathrm{CB}[8])$ in
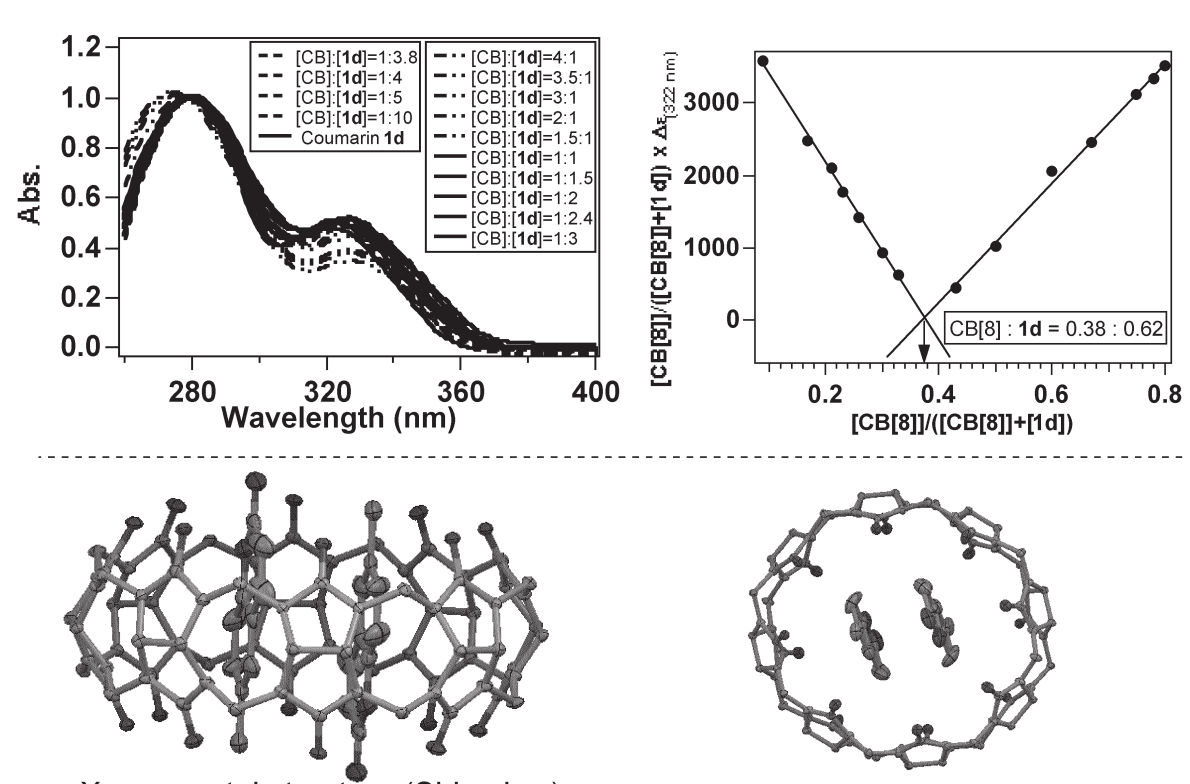

X-ray crystal structure (Side view)

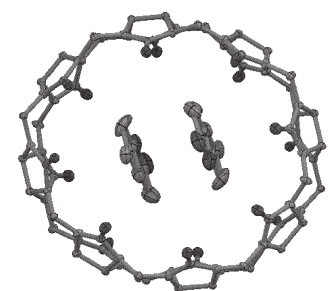

X-ray crystal structure (Top view)

Fig. 1. Characterization of HG complex of 1d@CB[8] by abs. spectroscopy/Job plot (top) and by single crystal XRD (bottom). altering the photoreactivity (Scheme 1) of coumarin derivatives. ${ }^{[29-33]}$ Our investigation revealed that both neutral and cationic coumarins 1a-k form stable HG complexes with CB[8]. ${ }^{[30,31]}$ The $\mathrm{HG}$ ratios of $\mathbf{1 a}-\mathbf{k}$ with $\mathrm{CB}[8]$ were examined using ${ }^{1} \mathrm{H}-\mathrm{NMR}$ and UV-Vis spectroscopy (Job plot) and by single crystal XRD (Fig. 1). [29-33]

Current investigations in our laboratory have established that the photoproduct resulting from templating of coumarins within $\mathrm{CB}[8]$ cavity is likely dictated by electrostatic/non-bonding interactions as well as by volume restrictions. ${ }^{[30,31]}$ In water/CB[8] media non-polar coumarins 1b-e preferentially formed syn-photoproducts, whereas polar coumarins with the ability to form $\mathrm{H}$-bonds as in $\mathbf{1} \mathbf{j}-\mathbf{k}$ preferentially formed the anti-dimer (Fig. 2, left). Based on spectroscopic characterization, computational modelling and control studies with coumarin substrates $(\mathbf{1 b}-\mathbf{i})$ we postulated that the $\mathrm{H}$-bonding interaction between the polar functional groups $\left(\mathrm{OH}\right.$ and $\left.\mathrm{NH}_{3}^{+}\right)$and the amide-carbonyl of $\mathrm{CB}$ portals is likely responsible for the observed selectivity (Fig. 2, left).

\subsection{Dynamic Supramolecular System in Solution}

Photoreactivity of non-polar coumarins $\mathbf{1 a}-\mathbf{i}$ within $\mathrm{CB}[8]$ provided an opportunity to enhance their photodimerization efficiency. Additionally, we observed that these non-polar coumarins formed dynamic HG complex in water (Fig. 2, right). For example, 1d formed a $\mathrm{H}: \mathrm{G}$ ratio of $1: 1.6$ indicating a mixture of $1: 1$ and $1: 2$ $\mathrm{CB}[8]: 1 d$ host-guest complex along with an uncomplexed coumarin in solution. The dimerization efficiency in the case of $\mathbf{1 d - e}$ was higher inside the cavity (Path A; Fig. 2, right), while for coumarins $\mathbf{1 a}, \mathbf{1 f}-\mathbf{i}$ the dimerization outside the cavity was much higher (Path B; Fig. 2, right). For example, the photodimerization of $\mathbf{1 d}$ is very effi- 
cient within the cavity viz., $50 \%$ conversion in $72 \mathrm{~h}$ in isotropic media (benzene) compared to $>70 \%$ conversion in $60 \mathrm{~min}$ in the presence of $\mathrm{CB}[8]$ in water. In general, the different stereoisomeric photoproducts are observed in the presence of $\mathrm{CB}$ [8] compared to isotropic media. For example, in the case of $\mathbf{1 d}$, syn dimers were observed within $\mathrm{CB}$ [8] and anti dimers in isotropic media. To understand the supramolecular system in detail we will concentrate on the photochemistry of $\mathbf{1 d}$ as a model chromophore to understand the influence of confinement within CBs.

\subsection{Photophysical Aspects - Effect of Confinement within CBs}

To ascertain the excited state chemistry of 1d within CB[8], we carried out steady state and time resolved emission fluorescence and phosphorescence measurements (Fig. 3). Photophysical investigations of 1d within $\mathrm{CB}[8]$ not only provided further insights into the nature of the reactive excited state, but also added credibility to our proposed dynamic behavior of $\mathrm{CB}[8]-\mathbf{1 d}$ host-guest complex in water. We observed (Fig. 3A) a $40 \mathrm{~nm}$ red shift, an increase in the emission quantum yield and an increase in the fluorescence lifetime (Fig. 3B) upon encapsulation of 1d within $\mathrm{CB}[8]$. The maximum emission intensity was observed for the 1:1 complex and the emission intensity decreased for the 1:2 complex because photodimerization pathway competes with the radiative pathway decreasing the emission quantum yield. Fluorescence lifetime measurements (Fig. 3B) showed three distinct decays with a long-lived component $(\sim 3.7 \mathrm{~ns})$ from a 1:1 HG complex, an $\sim 0.7$ ns component being the 1:2 HG complex and the $<0.1 \mathrm{~ns}$ being the uncomplexed form (1d is established ${ }^{[34]}$ to have a $<0.1$ ns component decay in water). In addition, there was also an observable phosphorescence at $77 \mathrm{~K}$ (Fig. 3C) in the presence of $\mathrm{CB}[8]$. Thus photophysical studies revealed that the photoreactions could occur from both the excited singlet and excited triplet state.

\subsection{Supramolecular Photocatalysis by Confinement within CB[8] Nano-cavity}

In principle, photochemical transformations with catalytic amounts of $\mathrm{CB}$ [8] are quite feasible, as our investigations have revealed that non-polar coumarins form dynamic host-guest (HG) complexes (Fig. 2; Path A) and react very efficiently in water. We employed $\mathbf{1 d}$ as a model system to investigate the feasibility of employing $\mathrm{CB}[8]$ as a catalytic supramolecular nano-reaction vessel. Our results point to a likely catalytic cycle (Fig. 4, left) involving $\mathrm{CB}[8]$ where the photodimerization of $\mathbf{1 d}$ results in the exclusive formation of syn-

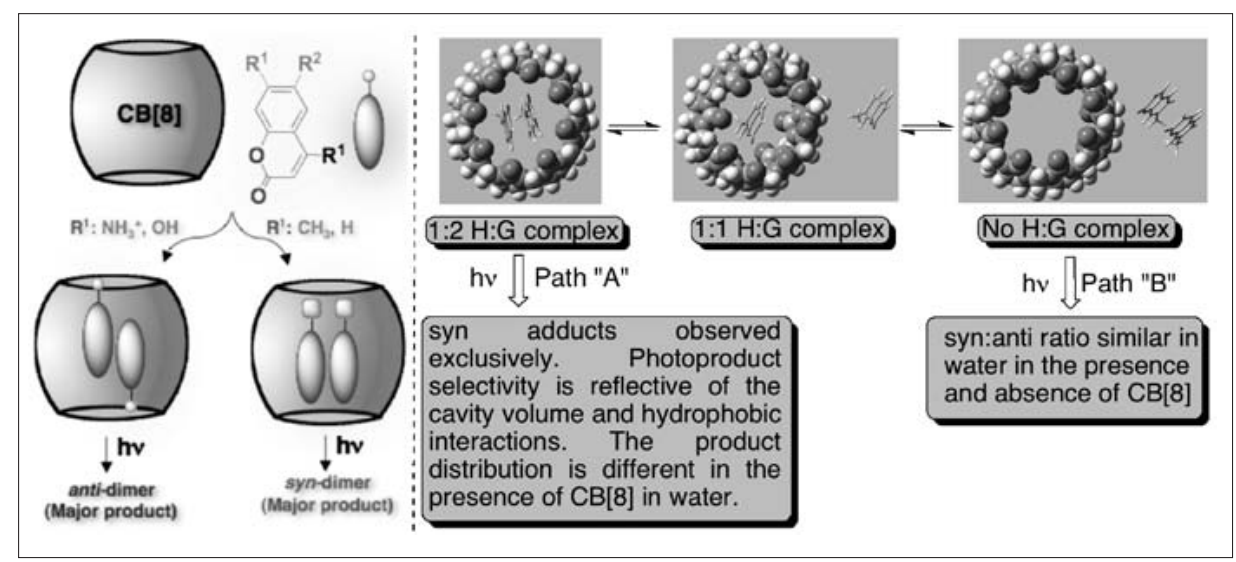

Fig. 2. Left: Photoproduct selectivity in polar and non-polar coumarins leading to anti- and syndimers respectively. Right: Host-guest dynamics and its influence on photochemical pathway within $\mathrm{CB}[8]$.

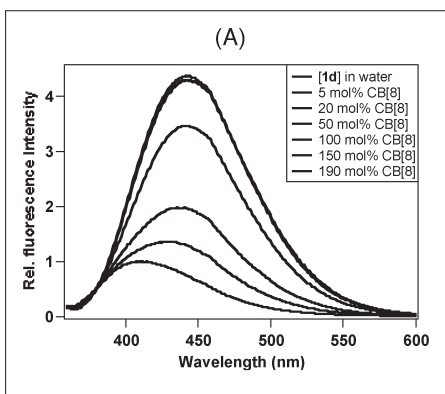

(B)

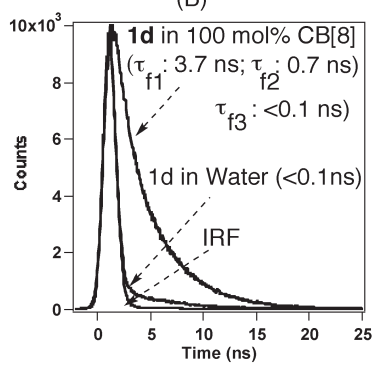

(C)

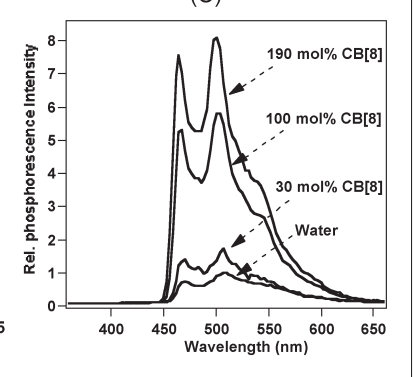

Fig. 3. Photophysical aspects of 6-methylcoumarin 1d in water. (A) Room temperature fluorescence, $(B)$ fluorescence lifetime at room temperature, $(C)$ phosphorescence at $77 \mathrm{~K}$.

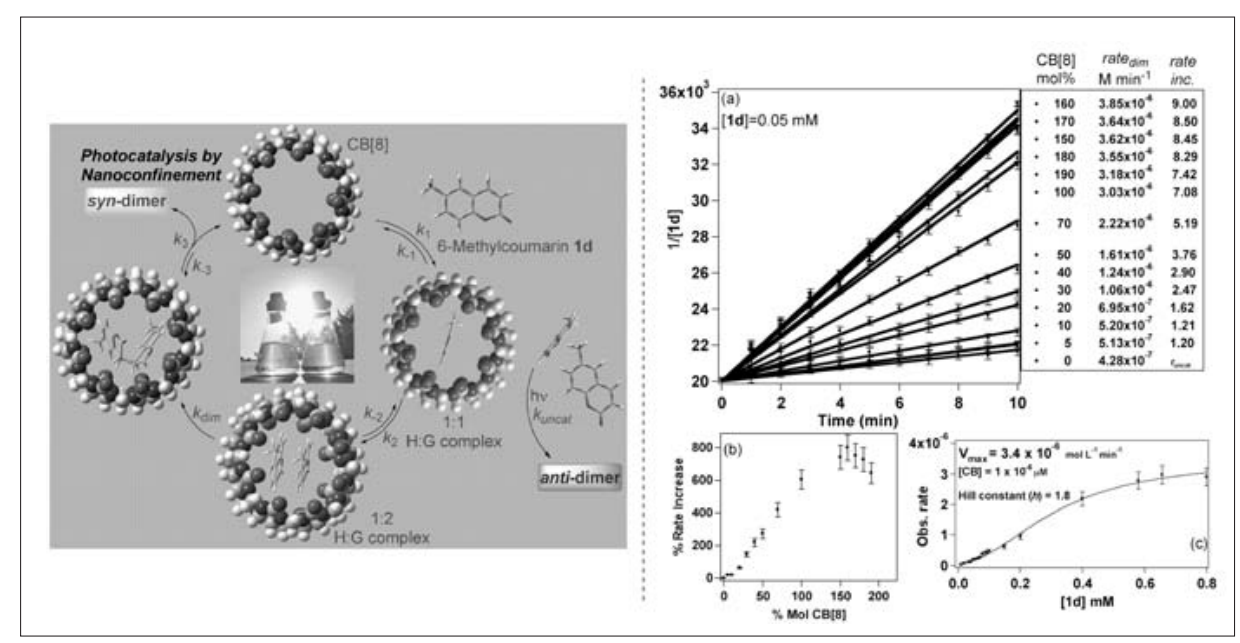

Fig. 4. Left: $\mathrm{CB}$ [8] mediated catalytic cycle for photodimerization of coumarins. Right: (a) Kinetics of photodimerization of $\mathbf{1 d}$ at various mol\% of $\mathrm{CB}[8]$; (b) Rate increase with various mol\% of $\mathrm{CB}[8]$. (c) Saturation kinetics; Error bars represent a 10\% error in the values.

dimers. A closer look at the catalytic cycle reveals that the photodimerization process occurs in the presence of catalytic amounts of $\mathrm{CB}[8]$ presumably due to the high photodimerization rate within the cavity. The photodimerization in water outside the $\mathrm{CB}[8]$ cavity (background reaction) determines the efficiency of the catalytic cycle and the selectivity in the photodimerization process (syn-anti ratio). The extent of background reaction compared to the reaction within the cavity can easily be un- derstood based on the photoproduct distribution as the anti dimer is formed outside the cavity (in water) while syn dimer is observed within the cavity.

In the present case, the anti-dimer is formed only outside the cavity whereas the syn isomers are formed within the cavity. Thus the ratios of syn-anti isomer not only reflect the selectivity within the confined environment of $\mathrm{CB}$ [8] but also the efficiency of the catalytic cycle. As the reaction in water has been postulated to occur from a 
triplet state, the background reaction can be completely quenched in the presence of oxygen. Thus, the catalytic efficiency can be enhanced by carrying out the reaction in $\mathrm{O}_{2}$ saturated solution as the photodimerization outside the cavity is lowered in the presence of $\mathrm{O}_{2}$ leading $\left(\mathrm{O}_{2}\right.$ acting as a triplet quencher) to a pronounced syn-anti ratio even at low mol\% of $\mathrm{CB}[8]$. Saturation kinetics (Fig. 4c) gave a turnover number of $3.4 \mathrm{~min}^{-1}$. The maximum rate of photodimerization (Fig. 4a,b) occurred at $160 \mathrm{~mol} \%$ of $\mathrm{CB}[8]$. This is a reflection of the dynamic nature of the system indicating that the maximum concentration of 1:2 HG complex occurs at $160 \mathrm{~mol} \%$ of $\mathrm{CB}[8]$. Additionally, the sigmoidal dependence on coumarin concentration (with constant $\mathrm{CB}[8]$ concentration) implies that the overall catalytic process is cooperative in nature (Fig. 4c). A Hill plot gave a Hill constant of 1.8 , ascertaining a positive allosteric effect. The positive allosterism could be envisioned based on the single crystal XRD of CB[8]-1d (Fig. 1) HG complex that showed a guest-induced shape change. ${ }^{[33]}$ The symmetrical $\mathrm{CB}[8]$ cavity becomes elliptical upon inclusion of the guest. We believe that the first coumarin binding with $\mathrm{CB}[8]$ induces a slight alteration of the shape of the cavity to accommodate the second coumarin facilitating the enhancement of photo-dimerization. This is reflected in the positive allosteric effect. We were successful in performing photodimerization of $\mathbf{1 d}$ with catalytic amounts of $\mathrm{CB}[8]$ under sunlight ( $8 \mathrm{~h}$ exposure) in water that resulted in complete conversion of 1d to syn photodimers highlighting a greener approach for synthetic manipulation. ${ }^{[29]}$

\subsection{Outlook}

Our results clearly show that cucurbiturils can be effectively employed in catalytic amounts to control light induced transformations. The effect of confinement and the enhanced reactivity within CBs is reflected in the photoproduct selectivity.

\section{Enantiospecific Photoreactions in Solution}

Asymmetric photoreactions have not enjoyed the same level of success as thermal reactions. Conventional chiral inductors employed in thermal reactions alter the relative activation energy in the ground state and therefore are not effective in inducing stereoselection during phototransformation. ${ }^{[35]}$ Chiral discrimination during phototransformation has to occur in the excited state within the short lifetime of the excited molecules/intermediates and/ or transition states. ${ }^{[35]}$ Photochemists have successfully employed various organized

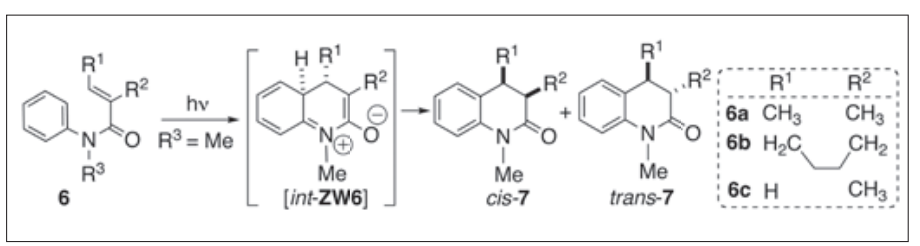

Scheme 2.

Conrotatory $6 \pi$-photocyclization of acrylanilides.

assemblies ${ }^{[36-41]}$ to carry out asymmetric photoreactions and have achieved varying degrees of success. To achieve stereoselection during phototransformation of prochiral reactants in solution, the chiral discrimination must transpire within the substrate, unlike in organized assemblies ${ }^{[36-41]}$ that provide the chiral discrimination, leading to noticeable stereoselectivity/specificity. Organized assemblies like crystals could provide a chiral environment if the prochiral substrate(s) crystallizes in one of the chiral space groups (molecular chirality), the process being inherently unpredictable. These molecularly chiral crystals could be transformed to chiral photoproducts with very high stereoselectivity/stereospecificity. ${ }^{[42-44]}$ It would be ideal to have a similar methodology of transferring molecular chirality from the reactant to point chirality in the product in solution during phototransformations. ${ }^{[45]}$

\subsection{Methodology}

Our approach is to make use of built-in molecular constraints within a reactant and transform them to chirally enriched photoproducts with high stereospecificity. The constraints make the reactants axially chiral and are based on the well-established concept of rotamer control via restricted bond rotation that has been successfully employed for various transformations. [8a,46-49] The methodology of employing axially chiral rotamers draws inspiration from Havinga's NEER principle (Non-Equilibrating Excited Rotamers), ${ }^{[50]}$ where conformer-based product control during photochemical reactions is well documented. The axially chiral chromophores were synthesized with relative ease using established literature procedures. ${ }^{[46-49]}$ Due to space limitations, we will detail the photochemistry of molecularly chiral (axially chiral) acrylanilides. ${ }^{[51-53]}$ We have also employed the above methodology to other photochemical reactions ( $\gamma$-hydrogen abstraction, $[2+2]$-cycloaddition, $4 \pi$-cyclization). ${ }^{[54-57]}$

\section{$2.26 \pi$-Photocyclization of Molecularly Chiral (Axially Chiral) Acrylanilides}

We chose to investigate $6 \pi$-photocyclization of molecularly chiral acrylanilides as model system to test our methodology because i) the photochemical pathway is well established in literature; ${ }^{[58-62]}$ ii) it is well known that bulky (tert-butyl) ortho substituents in N,N'-disubstituted anilides are molecularly chiral (axially chiral) due to restricted rotation of the $\mathrm{N}-\mathrm{C}($ Aryl) bond and are fairly stable at ambient conditions; iii) synthesis (three steps) and chromatographic separation are well documented in literature. ${ }^{[46-49]}$ Mechanistically, ${ }^{[58-62]} 6 \pi-$ photocyclization of achiral acrylanilides 6 (Scheme 2) upon direct irradiation occurs via a conrotatory ring closure (Scheme 2) from a singlet $\pi \pi^{*}$ excited state leading to the zwitterionic intermediate int-ZW6. ${ }^{[58-62}$ Depending on the substituents on the double bond ( $\mathrm{R}^{1}$ and/or $\mathrm{R}^{2}$ ), photocyclization of acrylanilides $\mathbf{6}$ yields a mixture of cis-7 and trans-7 3,4-dihydroquinolin-2one photoproducts (Scheme 2). The cis-: trans- $(c-7: t-7)$ ratio in the photoproduct was found to be dependent on the nature of the solvent employed (Table 1, entries 1719). It is well established in literature that in an aprotic solvent, $\mathrm{H}$-transfer to the zwitterionic intermediate formed from acrylanilides (without $o$-tert-butyl substituent) occurred to a large extent via a thermally allowed intramolecular suprafacial $[1,5]-\mathrm{H}$ shift, while in protic solvents or in the presence of Brønsted acids, the proton was delivered intermolecularly. ${ }^{[58-62]}$

\subsection{Regioselectivity in the $6 \pi$-Photocyclization of Molecularly Chiral Acrylanilides}

Atropisomeric mixtures of o-tert-butylacrylanilides with $\mathrm{N}$-methyl substitution $\mathbf{8 a}-\mathbf{g}$ were synthesized and irradiated using a $450 \mathrm{~W}$ medium pressure $\mathrm{Hg}$-lamp with Pyrex cutoff in various solvents (Table 1, Scheme 3). The photoproducts were characterized by NMR spectroscopy, ESI-MS and chromatographic analysis that confirmed the formation of cis-9 and trans-9 3,4-dihydroquinolin-2-ones without the tert-butyl group (Scheme 3, top). Irradiation of the corresponding N-H derivatives $\mathbf{1 0 a}-\mathbf{c}$ under identical conditions gave the cyclized products cis-11 and trans-11 (Scheme 3, middle), with the $o$ tert-butyl group intact on the phenyl ring. [58-62] It is striking to note that substitution at the amide nitrogen $(\mathrm{H} v s . \mathrm{Me})$ was able to dictate the regiochemistry of cyclization on the phenyl ring. Based on the behaviour of the parent acrylanilide 6 (Scheme 2 ), one would expect the cyclization of $\mathbf{8}$ to occur at the unsubstituted ortho-position on the phenyl ring and not at the ortho-carbon bearing the tert-butyl group, leading to cis-9 and trans-9 as observed. On the other 
Table 1. Enantioselectivity and cis/trans ratio during photocyclization ${ }^{a-c}$ of acrylanilides.

\begin{tabular}{|c|c|c|c|c|c|c|c|}
\hline Entry & Solvent & Substrate & $c-9: t-9$ & \multicolumn{2}{|c|}{ [\%] ee cis-9 } & \multicolumn{2}{|c|}{ [\%] ee trans-9 } \\
\hline \multicolumn{3}{|c|}{$\begin{array}{c}\alpha, \beta \text {-substitued axially chiral } \\
\text { acrylanilides }\end{array}$} & & $(+)-8$ & $(-)-8$ & $(+)-8$ & $(-)-8$ \\
\hline 1) & $1: 2$ THF- $\mathrm{C}_{6} \mathrm{H}_{6}$ & $8 a$ & $62: 38$ & $-e$ & $-{ }^{e}$ & $95(\mathrm{~A})$ & 89 (B) \\
\hline 2) & Acetone & & $55: 45$ & $-{ }^{e}$ & $-e$ & $94(\mathrm{~A})$ & 90 (B) \\
\hline 3) & $1: 2$ THF- $\mathrm{C}_{6} \mathrm{H}_{6}$ & $8 \mathbf{b}^{d}$ & $22: 78$ & $93(S, R)$ & $80(R, S)$ & $94(S, S)$ & - \\
\hline 4) & $\mathrm{MeOH}$ & & $65: 35$ & $99(S, R)$ & $85(R, S)$ & $99(S, S)$ & \\
\hline 5) & Acetone & & $67: 33$ & $92(S, R)$ & $92(R, S)$ & $88(S, S)$ & - \\
\hline 6) & $1: 2$ THF- $\mathrm{C}_{6} \mathrm{H}_{6}$ & $8 d$ & $42: 58$ & $-e$ & $-e$ & $88(A)$ & 91 (B) \\
\hline 7) & Acetone & & $46: 54$ & $-e$ & $-e$ & $91(\mathrm{~A})$ & 94 (B) \\
\hline 8) & $1: 2$ THF $\mathrm{C}_{6} \mathrm{H}_{6}$ & $8 e$ & $52: 48$ & 90 (B) & $91(\mathrm{~A})$ & 99 (B) & $93(A)$ \\
\hline 9) & $\mathrm{CHCl}_{3}$ & & $41: 59$ & $91(\mathrm{~B})$ & $98(A)$ & 95 (B) & $99(A)$ \\
\hline 10) & $\mathrm{MeOH}$ & & $70: 30$ & 99 (B) & $99(A)$ & 99 (B) & $99(A)$ \\
\hline 11) & Acetone & & $63: 37$ & 87 (B) & $90(A)$ & $90(\mathrm{~B})$ & $91(A)$ \\
\hline \multicolumn{3}{|c|}{$\begin{array}{l}\alpha \text {-substitued axially chiral } \\
\text { acrylanilides }\end{array}$} & \multicolumn{3}{|c|}{$(+)-8$} & \multicolumn{2}{|c|}{$(-)-8$} \\
\hline 12) & TFE & \multirow[t]{3}{*}{$8 f$} & $-f$ & \multicolumn{2}{|r|}{0} & \multicolumn{2}{|c|}{0} \\
\hline 13) & $1: 2$ THF- ${ }_{6} \mathrm{H}_{6}$ & & $-{ }^{f}$ & \multicolumn{2}{|r|}{0} & \multicolumn{2}{|c|}{0} \\
\hline 14) & Acetone & & $-f$ & \multicolumn{2}{|r|}{$92(\mathrm{~A})$} & \multicolumn{2}{|c|}{$94(\mathrm{~B})$} \\
\hline 15) & TFE & $8 g$ & $-f$ & \multicolumn{2}{|r|}{0} & \multicolumn{2}{|c|}{0} \\
\hline 16) & Acetone & & $-f$ & \multicolumn{2}{|r|}{$94(A)$} & \multicolumn{2}{|c|}{$92(\mathrm{~B})$} \\
\hline \multicolumn{8}{|c|}{ cis/trans ratio during photocyclization ${ }^{a-c}$ of achiral acrylanilides with $\mathbf{6} \mathbf{a}-\mathbf{b}$ and $\mathbf{1 0} \mathbf{a}-\mathbf{b}{ }^{h}$} \\
\hline Entry & Solvent & $\begin{array}{l}c-7 a: t-7 a \\
(\text { from } 6 a)^{g}\end{array}$ & $\begin{array}{l}c-7 b: t-7 b \\
(\text { from } 6 a)^{g}\end{array}$ & & $\begin{array}{l}-11 a: t-11 a \\
\text { from } 10 a)^{g}\end{array}$ & \multicolumn{2}{|c|}{$\begin{array}{l}c-11 b: t-11 b \\
{\text { from } 10 b)^{g}}^{g}\end{array}$} \\
\hline 17) & $1: 2$ THF- ${ }_{6} \mathrm{H}_{6}$ & $52: 48$ & $11: 89$ & & $72: 28$ & \multicolumn{2}{|c|}{ 82:18 } \\
\hline 18) & $\mathrm{CHCl}_{3}$ & $49: 51$ & $41: 59$ & & $70: 30$ & \multicolumn{2}{|c|}{$90: 10$} \\
\hline 19) & $\mathrm{MeOH}$ & 18:82 & 83:17 & & $80: 20$ & & - \\
\hline
\end{tabular}

alrradiations were performed with $450 \mathrm{~W}$ medium pressure $\mathrm{Hg}$-lamp under a constant flow of nitrogen (time varies based on the solvent between 90 min to $5 \mathrm{~h}$ ). Conversion varied between $5-70 \%$ depending on the solvent. Increasing the irradiation time $(>5 \mathrm{~h})$ resulted in higher conversion, but uncharacterized additional side products were observed. In acetone, conversion was $50-60 \%$. Isolated yields were 49 and $51 \%$ in acetone for 8 e and $8 f$ respectively. ${ }^{b} \mathrm{~A}$ and $\mathrm{B}$ refers to the first and second peak that elutes out on the HPLC for a given pair of enantiomers; values are average of 3 runs with $\pm 5 \%$ error; reaction temperature $0-3{ }^{\circ} \mathrm{C} ;(+)$ and $(-)$ represents the sign of their CD signals at $240 \mathrm{~nm}$ in methanol $(\mathbf{8 a}-\mathbf{c})$ and methylcyclohexane $(\mathbf{8 g})$. Similarly for $\mathbf{8 d}-\mathbf{f},(+)$ and $(-)$ represents the sign of optical rotation in $\mathrm{CHCl}_{3}$. Regis-(RR)-WHELK-01 chiral stationary phase employed for separation of cis and trans enantiomers. ${ }^{\circ}$ cis:trans Ratio and conversion based on relative integration of corresponding peaks in NMR and HPLC/GC. TFE=trifluoroethanol. ${ }^{d}$ Absolute configuration assigned based on comparison of optical rotation values from literature ( ref. [63]). In the case of $\mathbf{8 d}$ photoproducts, chromatographic separation is necessary prior to HPLC analysis as HPLC retention times of trans-8d overlaps with the reactant $\mathbf{8 d}$. ${ }^{\circ}$ cis-9 Enantiomers were not separable on chiral stationary phase employed in our laboratory. ${ }^{f}$ cis and trans Isomers not feasible in photoproducts from methacryloyl derivatives $\mathbf{8 c}, \mathbf{8 f}$ and $\mathbf{8 g}$ as they are $\alpha$-substituted derivatives. ${ }^{9}$ Photocyclization of various derivatives of parent acrylanilide $\mathbf{6 a}-\mathbf{b}$ already reported in literature (refs [58-62]). ${ }^{h}$ Only cis/trans selectivity was studied as the substrates are not axially chiral.

hand, in the corresponding $\mathrm{N}-\mathrm{H}$ derivatives 10, photocyclization occurred at the expected unsubstituted ortho carbon (similar to the parent acrylanilide 6). A mechanistic understanding for the difference in behaviour between the $\mathrm{N}$-methyl acrylanilide $\mathbf{8}$ and the corresponding N-H acrylanilide $\mathbf{1 0}$ is crucial if one is to employ the system for studying molecular chiral (axial chiral) transfer during phototransformations. ${ }^{[51-57]}$ tom). It should be emphasized that the rigid conformation in the solid state is taken as a starting point to rationalize the observed photobehavior in solution where dynamic movements (rotational, translational, etc.) are prevalent. Based on the analysis of the crystal structure, it is plausible that the conformation that is observed in the crystalline state (Scheme 3 bottom) is reflective of the diminished steric impediments between the $o$-tert-butyl and the N-Me groups. Examination of the crystal structure of axially chiral acrylanilides $8 f$ (Scheme 3 bottom) revealed that the dihedral angle is almost 90 degrees between the plane formed by the aromatic ring and the plane formed by the $\mathrm{N}$-methyl-amide carbonyl unit. The likely reason for this orientation is to minimize the 1,3-allylic strain ( $\mathrm{A}^{1,3}$-strain) between the ortho-tert-butyl and the N-methyl substituents. On the other hand, in the case of $\mathrm{N}-\mathrm{H}$ derivatives as in $\mathbf{1 0}$, the amide $\mathrm{N}-\mathrm{H}$ can tautomerize to the corresponding enol form in solution eliminating the A-strain (Scheme 3). The tautomerization of the N-H derivatives orient them in a conformation that is optimal for photocyclization at the unsubstituted ortho-carbon on the phenyl ring (Scheme 3, middle).

Based on the established mechanistic pathway for $6 \pi$-photocyclization, direct irradiation of $\mathrm{N}$-methyl substituted ortho-tertbutylacrylanilides $\mathbf{8}$ in methanol, TFE or 1:2 THF-benzene results in the singlet state reactivity. This likely results in a zwitterionic intermediate int-ZW8 to avoid the 1,3-allylic strain between the ortho-tert-butyl and $\mathrm{N}$-methyl substituents. The zwitterionic intermediate int-ZW8 subsequently undergoes either an $\mathrm{H}$ shift from the o-tert-butyl substituent or a $\mathrm{H}$-transfer from the protic solvent leading to $\operatorname{cis} \mathbf{- 9}$ and trans-9, with the loss of 2-methylpropene (Scheme 3). The intramolecular or intermolecular H-transfer depends on the solvent employed. ${ }^{[58-62]}$ Photocyclization in methanol- $d$ confirmed the $\mathrm{H}$-shift from protic solvents based on deuterium incorporation. ${ }^{[53]}$ On the other hand, $6 \pi$-photocyclization of the $\mathrm{N}-\mathrm{H}$ derivatives 10 likely occurs from the amide enol form that is oriented ideally for photocyclization at the unsubstituted ortho-carbon on the phenyl ring resulting in the zwitterionic intermediate int-ZW10 (Scheme 3). The zwitterionic intermediate int-ZW10 subsequently undergoes an intramolecular-shift or $\mathrm{H}$-shift from the solvent to cis-11 and trans-11. ${ }^{[58-62]}$ Thus, photocyclization of N-H ortho-tertbutyl acrylanilides $\mathbf{1 0}$ is similar to that reported for the parent acrylanilides 6 without an $o-{ }^{t} \mathrm{Bu}$ group (Scheme 2). ${ }^{[58-62]}$

\subsection{Enantiospecific $6 \pi$-Photocyclization of Molecularly Chiral Acrylanilides}

To ascertain the transfer of axial chirality to point chirality during $6 \pi$-photocyclization 


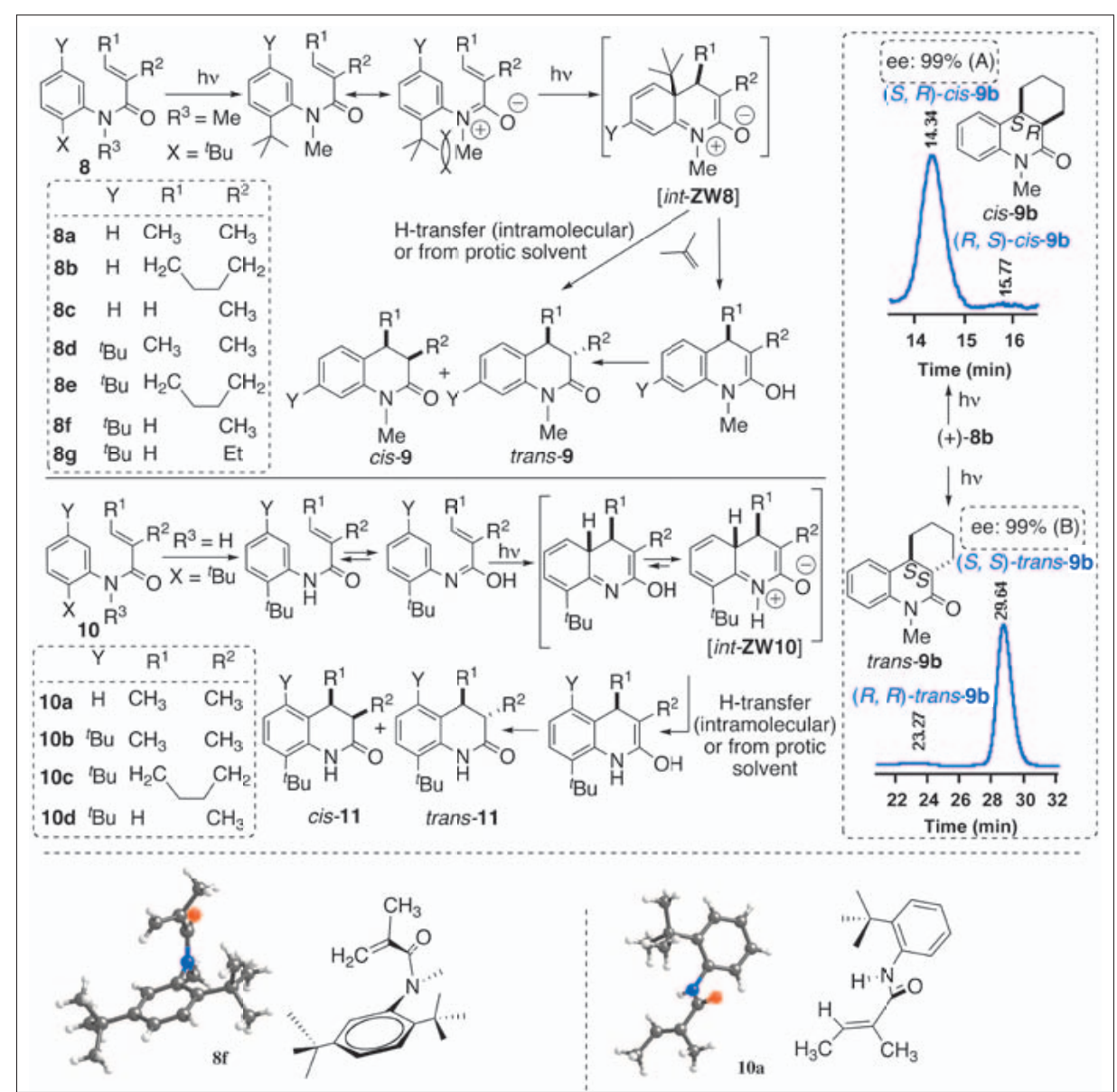

Scheme 3. 6r-photocyclization of axially chiral acrylanilides 8 (top) and NH-acrylanilides 10 (middle). Bottom: X-ray crystal structure of $\mathbf{8 f}$ (left), and 10a (right). Insert: HPLC analysis of cisand trans-photoproducts upon irradiation of $(+) 8 \mathbf{b}$.

molecularly chiral acrylanilides $\mathbf{8}$, we examined the enantiomeric excess in the 3,4-dihydroquinolin-2-ones photoproducts cis-9 and trans-9 (Table 1). In the case of $\alpha, \beta$-substituted axially chiral $\mathbf{8 a}-\mathbf{b}$ and $\mathbf{8 d}-$ e, very high enantiomeric excess ( $90 \%)$ was observed in photoproducts in both direct irradiation (in solvents methanol, $\mathrm{CHCl}_{3}$ and 1:2 THF-C $\mathrm{H}_{6}$ ) and sensitized irradiations (acetone as solvent and sensitizer). The optical antipodes of $\mathbf{1}$ gave the opposite enantiomers in the photocyclized product indicating that the system was well behaved (Table 1; entries 1-11).

Based on our mechanistic analysis we postulated that the photocyclization occurred at the ortho carbon via 'int-ZW8' (Scheme 3) with the eventual loss of the $o$-tert-butyl substituent. If this holds true, the enantiomeric excess in the photoproducts (cis-9 and trans-9) must be identical, as the resulting zwitterionic intermediate 'int-ZW8' (Scheme 3) has a defined chiral center at the benzylic position formed by stereospecific ring closure. The second proton transfer step is non-stereospecific leading to $\mathrm{cis}$ and trans photoproducts with identical ee values. Fortunately, we were successful in separating the enantiomers of both the cis-9b and the trans-9b in the case of cyclohexyl derivatives $\mathbf{8 b}$ (Scheme
3; HPLC insert). Inspection of Table 1 indicates that similar enantiomeric excess was observed in both cis-9 and trans-9 photoproducts. Additionally, the benzylic carbon in the cis and trans photoproducts has the same absolute stereochemistry adding credibility to our proposed mechanism (Scheme 3; HPLC insert). For example in the case of $(+)-8 \mathbf{b}$, photocyclization in methanol gives $(S, R)$-cis-9b and $(S, S)$-trans-9b with the same absolute stereochemistry $(S)$ at the benzylic position.

Inspection of Table 1 reveals that the $\beta$-substituent in the alkene is crucial for achieving the high enantiomeric excess under direct irradiation conditions (singlet spin state reactivity). For example, in 1:2 THF- $\mathrm{C}_{6} \mathrm{H}_{6}$ as solvent the $e e$ value of $>90 \%$ was observed for (+)-8d with $\beta-\mathrm{CH}_{3}$ substituent, where as $0 \%$ ee value is observed for the corresponding methacryloyl derivative (+)-8f with $\beta$-H substituent (Table 1; compare entries 6 and 13).

\subsection{Reactive Spin-state Dependent Enantiospecific 6r-Photocyclization of Axially Chiral Acrylanilides}

While the excited singlet-state reactivity (via direct irradiation) led to a racemic mixture in the photoproducts for $\alpha$-substituted axially chiral acrylanilides $\mathbf{8 f}$ and $\mathbf{8 g}$, the corresponding triplet reactivity (via triplet sensitization with acetone acting as the solvent and sensitizer) led to enantiomeric ratios (e.r. values) $>95: 05$ in the 3,4-dihydroquinolin-2-one photoproduct $\mathbf{9}$ at ambient conditions. To examine the role of reactive spin state $\left(\mathrm{S}_{1}\right.$ or $\left.\mathrm{T}_{1}\right)$ dependent photocyclization of axially chiral $\alpha$-substituted acrylanilides 8f-g (8f: $\alpha$-methyl and 8g: $\alpha$-ethyl) to the corresponding 3,4-dihydroquinolin-2-one photoproduct 9, we carried out detailed photophysical studies with $\alpha$-substituted axially chiral substrates $\mathbf{8 f}-\mathbf{g}$ and the $\mathrm{N}-\mathrm{H}$ $o$-tert-butyl derivative $\mathbf{1 0 d}$ and $\mathrm{N}-\mathrm{Me}$ derivative $\mathbf{6 c}$ without the $o$-tert-butyl group. Both 8f-g showed fluorescence (Fig. 5A) at room temperature in methylcyclohexane $(\mathrm{MCH})$. The emission maxima shifted bathochromically upon changing the solvent from non-polar $\mathrm{MCH}$ to polar solvents like ethanol or acetonitrile, similar to the fluorescence emission behaviour of other methacrylanilides that are reported in literature. ${ }^{[59]}$ Additionally, for $\mathbf{8 f}-\mathbf{g}$ we observed phosphorescence (Fig. 5A) at $77 \mathrm{~K}$ in $\mathrm{MCH}$ glass with a triplet energy $\left(\mathrm{E}_{\mathrm{T}}\right)$ of $\sim 77.3 \mathrm{kcal} \cdot \mathrm{mol}^{-1}$ and a lifetime $\left(\tau_{\mathrm{p}}\right)$ of $\sim 1.58$ s (Fig. $5 \mathrm{~B}$ ). Based on the emission studies, ${ }^{[64]}$ it is clear that the lowest excited singlet and triplet state have a $\pi \pi^{*}$ configuration in $\mathbf{8 f}-\mathbf{g}$.

Based on the established paradigm ${ }^{[64]}$ for photochemical reactions viz photocyclization, a zwitterionic intermediate is expected for the photocyclization from the $\pi \pi^{*}$ singlet excited-state $S_{1}\left(\pi \pi^{*}\right)$ and a diradicaloid intermediate is likely from the corresponding $\pi \pi^{*}$ triplet excited-state $\mathrm{T}_{1}\left(\pi \pi^{*}\right){ }^{[64]}$ This prompted Ogata and coworkers ${ }^{[59,60]}$ to propose a zwitterionic intermediate originating from the singlet $\pi \pi^{*}\left(S_{1} \pi \pi^{*}\right)$ excited-state for the $6 \pi$-photocyclization of achiral acrylanilide (N-H substituted derivative without ortho-tertbutyl group on the phenyl ring). In the case of $\mathbf{8 f}-\mathbf{g}$, we believe that the restricted $\mathrm{N}_{-}$ $\mathrm{C}$ (aryl) bond rotation not only imparts axial chirality (molecular chirality) to the system but also enables us to access the triplet state (as we observe phosphorescence) at $77 \mathrm{~K}$ in $\mathrm{MCH}$ glass. While in the case of achiral N-H acrylanilide (viz., ortho-tertbutyl N-H acrylanilide 10d) we failed to observe any phosphorescence at $77 \mathrm{~K}$, both achiral and axially chiral N-methyl derivatives 8f-g (molecular chiral $\mathbf{8 f}-\mathbf{g}$ and achiral 6c) gave observable phosphorescence at $77 \mathrm{~K}$ in $\mathrm{MCH}$ glass. Thus the presence of N-Me substituent is crucial to accessing the triplet-excited manifold. We believe that the enolization of the N-H acrylanilides (as in 10d; Fig. 5C) to the enol form enables it to cyclize only from the singletexcited state, as it is oriented optimally for $6 \pi$-photocyclization. On the other hand, 


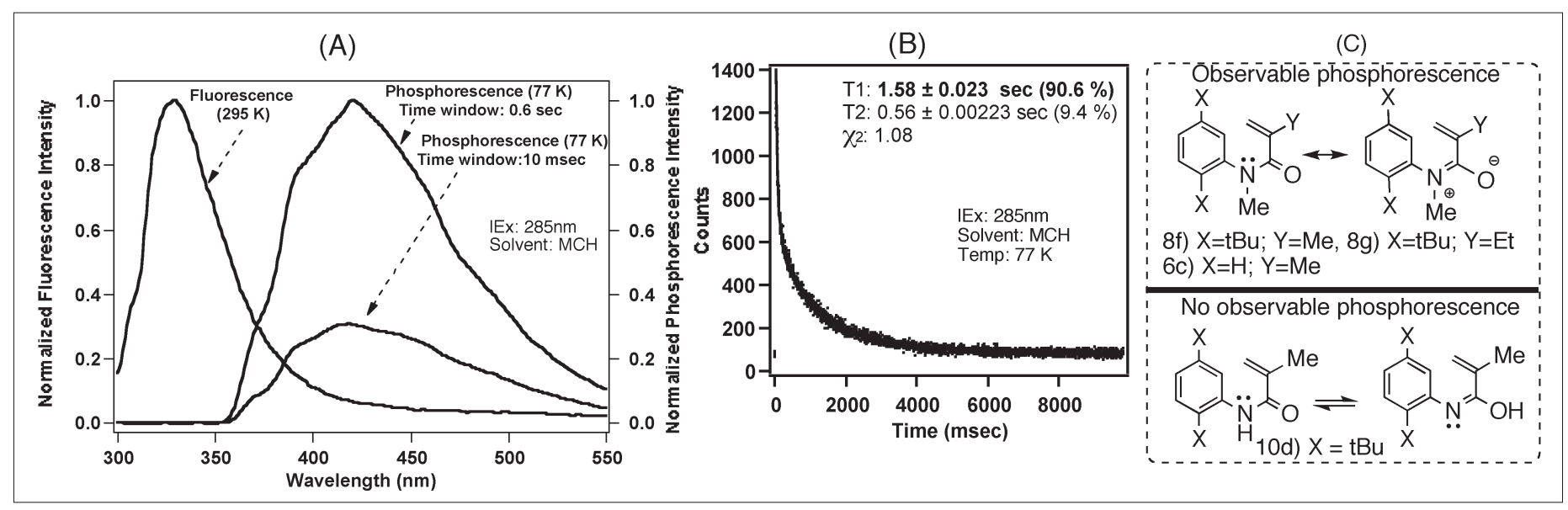

Fig. 5. (A) Fluorescence (at $298 \mathrm{~K}$ ) and phosphorescence (at $77 \mathrm{~K}$ ) of $8 \mathbf{f}$ in methylcyclohexane (MCH). (B) Phosphorescence decay kinetics of $8 \mathrm{ff}$ at 77 $\mathrm{K}$ in $\mathrm{MCH}$ glass. (C) Role of $\mathrm{N}$-methyl (8f-g and $\mathbf{6 c}$ ) and N-H substitution (10d).

in case of the $\mathrm{N}$-methyl derivatives (as in $\mathbf{8 f}-\mathbf{g}$; Fig. 5C), the nitrogen lone pair is part of the $6 \pi$-backbone leading to photoreactivity from the $\pi \pi^{*}$ excited state. Based on the phosphorescence, the triplet $\pi \pi^{*}$ state of $\mathbf{8 f}-\mathbf{g}$ lies at $\sim 77 \mathrm{kcal} \bullet \mathrm{mol}^{-1}$. Hence triplet energy transfer from acetone $\left(\mathrm{E}_{\mathrm{T}} \sim 79\right.$ $\left.\mathrm{kcal} \cdot \mathrm{mol}^{-1}\right)^{[65]}$ is quite likely. The involvement of triplet spin state viz., $\mathrm{T}_{1}\left(\pi \pi^{*}\right)$ in the reaction pathway was ascertained by carrying out the reaction under $\mathrm{O}_{2}$ saturated conditions that resulted in $<5 \%$ conversion. ${ }^{[53]}$ Thus $6 \pi$-photocyclization of $\mathbf{8 f}-\mathbf{g}$ could possibly occur from either the singlet $\left(\mathrm{S}_{1}\right)$ or the triplet $\left(\mathrm{T}_{1}\right)$ spin-state depending on the reaction conditions. ${ }^{[66]}$

Similar to the photocyclization (direct irradiation) of $\alpha, \beta$-substituted axially chiral acrylanilides that occurred via zwitterionic intermediate 'int-ZW8' (Scheme 3), direct irradiation (singlet chemistry) in the case of ortho-tert-butyl $\alpha$-substituted axially chiral acrylanilides $\mathbf{8 f}-\mathbf{g}$ led to photocyclization that occurred at the ortho carbon via an analogous zwitterionic intermediate 'int-ZW8fg' (Scheme 4, top) followed by a non-stereospecific hydrogen migration with the eventual loss of the o-tert-butyl substituent. As the photocyclization occurred from the $S_{1}\left(\pi \pi^{*}\right)$ excited state upon direct irradiation in the case of $\mathbf{8 f}-\mathbf{g}$, the formation of a zwitterionic intermediate ('int-ZW8fg') was expected.[58-62] Similarly, triplet sensitized irradiation of $\mathbf{8 f}-\mathbf{g}$ led to photocyclization from $\mathrm{T}_{1}\left(\pi \pi^{*}\right)$ excited state resulting in a diradical (triplet diradical) intermediate 'int-DR8fg' (Scheme 4, bottom). This triplet diradical 'int-DR8fg' subsequently abstracts a hydrogen atom from o-tert-butyl substituent leading to 3,4-dihydroquinolin-2-one photoproduct 9. The high enantiomeric ratio (Table 1) in the photoproduct $\mathbf{2}$ under sensitized irradiation (acetone as solvent and sensitizer) points out to a stereospecific hydrogen abstraction via a cyclic six-membered transition (Scheme 4, bottom) state from triplet diradical intermediate (int-DR8fg).

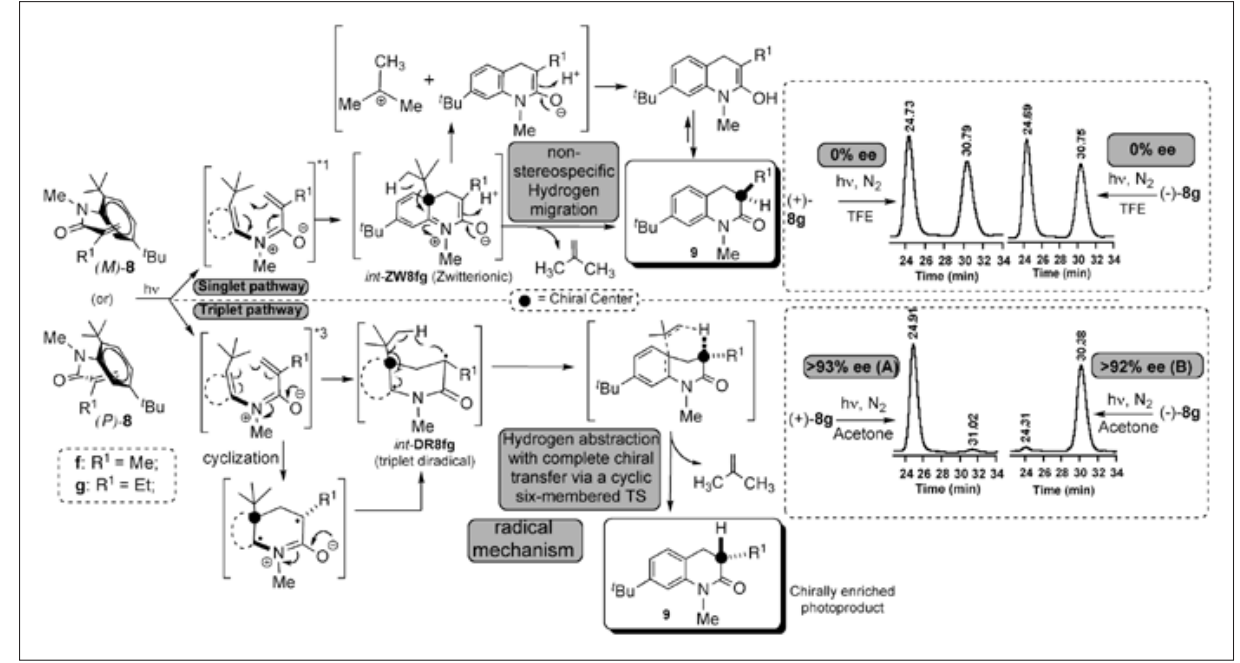

Scheme 4. Photocyclization of axially chiral $\alpha$-substituted acrylanilides $\mathbf{8} \mathbf{f}-\mathbf{g}$ and under direct (singlet) and sensitized (triplet) irradiations.

Thus the excited spin state $\mathrm{S}_{1}\left(\pi \pi^{*}\right)$ or $\mathrm{T}_{1}$ $\left(\pi \pi^{*}\right)$ not only leads to the formation of same photoproduct via two different reactive intermediates and/or transition states, but also determines the e.r. values in the photoproduct in the case of $\alpha$-substituted axially chiral acrylanilides $\mathbf{8 f}-\mathbf{g}$.

\subsection{Outlook}

Our strategy of employing axially chiral chromophores has opened up the possibility of achieving very high enantiomeric excess in phototransformations in solution, a traditionally difficult task. Employing axially chiral chromophores that equilibrate very slowly in the ground state leading to very high enantioselectivity in the photoproducts draws inspiration from Havinga's NEER principle (NonEquilibrating Excited Rotamers), where conformer-based product control is well documented. ${ }^{[50]}$ We have also been very successful in employing this methodology of using axially chiral chromophores in various photochemical transformations. Axially chiral chromophores in conjunc- tion with their corresponding achiral counterparts offer rich and divergent photoreactivity that helps us to better understand mechanisms of light-induced stereospecific transformations.

\section{Conclusion}

The ongoing investigations in our lab have uncovered two methodologies to achieve high selectivity in solution during photochemical transformations. Using a supramolecular approach, we have employed cucurbiturils in catalytic amounts to control selectivity during photochemical transformations. To tackle the traditional challenge of controlling enantioselectivity we have employed axially chiral motifs to enantiospecifically transfer the axial chirality in the reactant to point chirality in the photoproduct. This methodology was found to be efficient for various photoreactions thus presenting itself as a generalized methodology to perform light-induced transformations in solution at ambient conditions. 


\section{Acknowledgment}

The authors thank the Swiss Chemical Society for the 2010 SCS GrammaticakisNeumann Prize and for the hospitality during the award ceremony at ETH-Zürich. The work was performed with generous funding from the National Science Foundation (NSFCAREER: CHE-0748525). The authors thank the NSF ND-EPSCoR program for a doctoral dissertation fellowship to BCP (Grant EPS0814442). AJA thanks a Graduate Student Fellowship from NSF and UNCF/MERCK science initiative research grant. The start-up fund to establish the research program was with generous funding from the Department of Chemistry and Biochemistry, North Dakota State University, Fargo ND. The authors also thank the ND-EPSCoR program for seed grants (2006-2007: Grant EPS-0447679 and 20102011: Grant EPS-0814442). The authors would like to thank Dr. Nilotpal Barooah and Dr. Josepha Jesuraj for their contributions.

Received: January 25, 2011

[1] N. J. Turro, Proc. Natl. Acad. Sci. USA 2005, 102, 10766.

[2] 'Artificial Enzymes', Ed. R. Breslow, WileyVCH, Weinheim, 2005.

[3] I. Tabushi, Acc. Chem. Res. 1982, 15, 66.

[4] S. A. Nepogodiev, J. F. Stoddart, J. Am. Chem. Soc. 1998, 98, 1959

[5] R. Behrend, E. Meyer, F. Rusche, Liebigs Ann. Chem. 1905, 339, 1 .

[6] W. A. Freeman, W. L. Mock, N.-Y. Shih, J. Am. Chem. Soc. 1981, 103, 7367.

[7] J. W. Lee, S. Samal, N. Selvapalam, H.-J. Kim, K. Kim, Acc. Chem. Res. 2003, 36, 621.

[8] W. S. Jeon, K. Moon, S. H. Park, H. Chun, Y. H. Ko, J. Y. Lee, E. S. Lee, S. Samal, N. Selvapalam, M. V. Rekharsky, V. Sindelar, D. Sobransingh, Y. Inoue, A. E. Kaifer, K. Kim, $J$. Am. Chem. Soc. 2005, 127, 12984.

[9] A. Day, A. P. Arnold, R. J. Blanch, B. Snushall, J. Org. Chem. 2001, 66, 8094.

[10] J. Lagona, J. C. Fettinger, L. Isaacs, J. Org. Chem. 2005, 70, 10381 .

[11] W. Ong, M. G.-. Kaifer, A. E. Kaifer, Org. Lett. 2002, 4, 1791.

[12] C. Klock, R. N. Dsouza, W. M. Nau, Org. Lett. 2009, 11, 2595.

[13] J. Rebek, Acc. Chem. Res. 1990, 23, 399

[14] C. L. D. Gibb, A. K. Sundaresan, V. Ramamurthy, B. C. Gibb, J. Am. Chem. Soc. 2008, 130,4069

[15] W. L. Mock, T. A. Irra, J. P. Wepsiec, T. L. Manimaran, J. Org. Chem. 1983, 48, 3619.
[16] W. L. Mock, T. A. Irra, J. P. Wepsiec, M. Adhya, J. Org. Chem. 1989, 54, 5302.

[17] J. Kang, G. Hilmersson, J. Santamaria, J. J. Rebek, J. Am. Chem. Soc. 1998, 120, 3650.

[18] 'Photochemistry in Organized and Constrained Media', Ed. V. Ramamurthy, Wiley-VCH, New York, 1991.

[19] R. Wang, L. Yuan, D. H. Macartney, J. Org. Chem. 2006, 71, 1237.

[20] J. N. Moorthy, K. Venkatesan, R. G. Weiss, J. Org. Chem. 1992, 57, 3292.

[21] G. S. Hammond, C. A. Stout, A. A. Lamola, $J$. Am. Chem. Soc. 1964, 86, 3103.

[22] R. Hoffman, P. Wells, H. Morrison, J. Org. Chem. 1971, 36, 102.

[23] K. Muthuramu, V. Ramamurthy, J. Org. Chem. 1982, 47, 3976.

[24] W. W. Mantulin, P.-S. Song, J. Am. Chem. Soc. 1973, 95, 5122.

[25] S. S. J. de Melo, R. S. Becker, A. L. Maqanita, J. Phys. Chem. 1994, 98, 6054

[26] R. S. Becker, S. Chakravorti, C. A. Gartner, M. d. G. Miguel, J. Chem. Soc. Faraday Trans. 1993, 89, 1007

[27] G. F. Fedorin, V. P. Georgievskii, J. Appl. Spectros. 1974, 20, 122

[28] S. K. Allen, A. Todd, J. M. Allen, Biochem. Biophys. Res. Commun. 1997, 235, 615.

[29] B. C. Pemberton, N. Barooah, D. K. Srivatsava, J. Sivaguru, Chem. Commun. 2010, 46, 225.

[30] N. Barooah, B. Pemberton, A. C. Johnson, J. Sivaguru, Photochem. Photobiol. Sci. 2008, 7 , 1473 .

[31] N. Barooah, B. Pemberton, J. Sivaguru, Org. Lett. 2008, 10, 3339.

[32] B. Pemberton, E. Kumarasamy, S. Jockusch, D. K. Srivatsava, J. Sivaguru, Can. J. Chem. 2011, DOI: $10.1139 / \mathrm{v} 10$

[33] B. C. Pemberton, R. K. Singh, S. Jockusch, J. P. Da Silva, A. Ugrinov, N. J. Turro, D. K. Srivatsava, J. Sivaguru, Chem. Commun. 2011, submitted.

[34] T. Wolff, H. Goerner, Phys. Chem. Chem. Phys. 2004, 6, 368.

[35] Y. Inoue, in 'Chiral Photochemistry', Eds. Y. Inoue, V. Ramamurthy, Marcel Dekker, New York, 2004, pp. 129.

[36] V. Ramamurthy, in 'Photochemistry in Organized and Constrained Media', WileyVCH, New York, 1991, p. 429.

[37] N. J. Turro, M. Garcia-Garibay, in 'Photochemistry in Organized and Constrained Media', Ed. V. Ramamurthy, Wiley-VCH, New York, 1991, p. 1.

[38] G. M. J. Schmidt, Pure Appl. Chem. 1971, 27, 647.

[39] J. N. Gamlin, R. Jones, M. Leibovitch, B. Patrick, J. R. Scheffer, J. Trotter, Acc. Chem. Res. 1996, 29, 203.

[40] T. Mori, R. G. Weiss, Y. Inoue, J. Am. Chem. Soc. 2004, 126, 8961
[41] A. Bauer, F. Westkämper, S. Grimme, T. Bach, Nature 2005, 436, 1139.

[42] F. Toda, Acc. Chem. Res. 1995, 28, 480

[43] M. Sakamoto, M. Kato, Y. Aida, K. Fujita, T. Mino, T. Fujita, J. Am. Chem. Soc. 2008, 130 1132.

[44] M. Veerman, M. J. E. Resendiz, M. A. GarciaGaribay, Org. Lett. 2006, 8, 2615.

[45] T. Bach, J. Schröder, K. Harms, Tetrahedron Lett. 1999, 40, 9003.

[46] D. P. Curran, H. Qi, S. J. Geib, N. C. DeMello, J. Am. Chem. Soc. 1994, 116, 3131

[47] D. P. Curran, G. R. Hale, S. J. Geib, A. Balog, Q. B. 1. Cass, A. L. G. Degani, M. Z. Hernandes, L. C. G. Freitas, Tetrahedron: Asymm. 1997, 8 , 3955.

[48] J. Clayden, Chem. Commun. 2004, 127.

[49] A. Honda, K. M. Waltz, P. J. Carroll, P. J. Walsh, Chirality 2003, 15, 615.

[50] E. Havinga, J. L. M. A. Schlatmann, Tetrahedron 1961, 16, 146

[51] A. J.-L. Ayitou, S. J., J. Am. Chem. Soc. 2009 , $131,5036$.

[52] A. J.-L. Ayitou, J. Sivaguru, Chem. Commun 2010, ASAP. DOI: $10.1039 / C 0 C C 04416 D$.

[53] A. J.-L. Ayitou, J. Sivaguru, A. Ugrinov, Photochem. Photobiol. Sci. 2009, 8, 751.

[54] A. J.-L. Ayitou, J. L. Jesuraj, N. Barooah, A. Ugrinov, J. Sivaguru, J. Am. Chem. Soc. 2009, 131,11314

[55] J. L. Jesuraj, J. Sivaguru, Chem. Commun. 2010, 46, 4791.

[56] N. Vallavoju, J. Sivaguru, unpublished results.

[57] E. Kumarasamy, J. Sivaguru, unpublished results.

[58] O. L. Chapman, W. R. Adams, J. Am. Chem. Soc. 1968, 90, 2333.

[59] Y. Ogata, K. Takagi, I. Ishino, J. Org. Chem. 1971, 36, 3975.

[60] I. Ninomiya, S. Yamauchi, T. Kiguschi, A Shinobara, T. Naito, J. Chem. Soc., Perkin Trans. 1 1974, 1747.

[61] T. Bach, B. Grosch, T. Strassner, E. Herdtweck, J. Org. Chem. 2003, 68, 1107.

[62] G. R. Lenz, J. Org. Chem. 1976, 41, 2201.

[63] T. Naito, Y. Tada, I. Ninomiya, Heterocycles 1984, 22, 237.

[64] N. J. Turro, 'Modern Molecular Photochemistry', University Science Books, New York, 1991, 228-230 and 264-266.

[65] S. L. Murov, I. Carmichael, G. L. Hug, 'Handbook of Photochemistry', Marcel Dekker, New York, 1993, 54-98.

[66] R. Lapouyade, C. Manigand, A. Nourmamod, Can. J. Chem. 1985, 63, 2192. 